\title{
Synchrotron X-ray Micro Tomography at the Advanced Light Source: In-Situ Sample Environments for Advanced Aerospace Materials
}

\author{
$\underline{\text { Harold S. Barnard }}^{1 *}$, A.A Macdowell ${ }^{1}$, D.Y.Parkinson ${ }^{1}$, N.M.Larson ${ }^{1,2}$, J.C.Peterson ${ }^{1}$ \\ F.Panerai $^{3}$, N.N.Mansour ${ }^{3}$, Y.Gao $^{4}$ \\ 1. Advanced Light Source, Lawrence Berkeley National Laboratory, Berkeley CA, USA. \\ 2. University of California, Santa Barbara, Dept. of Materials Science, Santa Barbara CA, USA. \\ 3. NASA Ames Research Center, Moffett Field CA, USA. \\ 4. GE Global Research, Niskayuna NY, USA \\ * Corresponding author, hbar@lbl.gov
}

\section{Introduction}

The Advanced Light Source (ALS) at Lawrence Berkeley National Lab is a 3rd generation synchrotron X-ray source devoted to basic and applied sciences. Beamline 8.3.2 at the ALS performs micro computed tomography $(\mu \mathrm{CT})$ analysis of materials, providing 3D imaging of materials with high resolution $(0.6 \mu \mathrm{m} / \mathrm{voxel})$. The $\mu \mathrm{CT}$ technique is regularly applied to a diverse range of materials ranging from aerospace to geology to plant biology, and provides valuable, non-destructive characterization of the material's internal micro structure. The high flux hard x-rays provided by the synchrotron source enables $\mu \mathrm{CT}$ imaging with relatively short data acquisition times ( $\sim 1$ second to $100 \mathrm{~s}$ of seconds per $\mu \mathrm{CT}$ scan). This speed allows for real-time in-situ analysis of the microstructural evolution of materials under realistic conditions provided by environmental test cells. These conditions can include high temperature, pressure, and mechanical loading in a controlled inert or reactive atmosphere or in vacuum. These conditions can be combined in multi-parameter trajectories to model complex dynamic processes such as atmospheric re-entry of spacecraft or fabrication of advanced composites. Recent advances in in-situ high temperature $\left(>1200^{\circ} \mathrm{C}\right)$ mechanical testing, in-situ high temperature $\left(\sim 1800^{\circ} \mathrm{C}\right)$ processing of ceramics and composites will be presented with examples from aerospace materials science.

\section{Advanced Aerospace Materials}

Advanced materials that maintain their strength at high temperature are an active area of research and are critically important for the advancement of future aerospace technology. Two categories of materials will be discussed. 1) Ceramic matrix composites (CMC) - typically a silicon carbide ( $\mathrm{SiC}$ ) matrix reinforced by $\mathrm{SiC}$ or carbon fibers - are a promising class of light weight structural material targeted to withstand operational temperatures approaching $1500^{\circ} \mathrm{C}$ and were recently deployed in commercial jet engines [1,2,3]. 2) Ablative heatshield materials for spacecraft - typically porous carbon fiber foams or woven carbon fiber impregnated with phenolic resin - are critical for high velocity entry into the atmosphere of Earth or other planets [4,5]. The performance of these materials depends strongly on the details of how their microstructure forms during fabrication and how it evolves under extreme operational loads and environmental conditions.

\section{In-situ Materials Testing}

In-situ analysis of advanced materials using $\mu \mathrm{CT}$ under realistic operational loads, temperatures, and environments provides essential information about how the material's microstructure evolves. The 3D data generated by $\mu \mathrm{CT}$ is typically used for quantitative assessment of morphological changes (e.g. porosity, crack propagation, etc.) or provide inputs for or validation of complex multi-physics materials simulations. In-situ high temperature mechanical testing has therefore become a major part of the $\mu \mathrm{CT}$ program at the ALS with capabilities that have expanded to include uniaxial tension and compression (up to $2000 \mathrm{~N}$ loads), three point bending, and four point bending at temperatures up to $1500^{\circ} \mathrm{C}[4,5,6]$.

A fundamental understanding of CMC fabrication processes including sintering, reactive melts of ceramic constituents, and polymer pyrolysis is becoming increasingly important for further development of CMCs. Similarly, observation of the microstructural effects of high temperature on chemistry, gas flow, and permeability 
are necessary to couple with simulation for predictive modeling of heatshields for NASA's Orion Spacecraft program and future Mars missions such as the Mars Sample Return program [4.5]. To address this scientific need, a new in-situ tube furnace and environmental cell have been developed at the ALS to provide stable sample temperature up to $1800^{\circ} \mathrm{C}$ with a sealed sample cell providing prescribed atmospheric conditions. The system consists of a $1 \mathrm{~kW}$ tube furnace with a $6 \times 6 \mathrm{~mm}$ beam port, and a vertical $50 \mathrm{~cm}$ diameter bore heated by a $\mathrm{MoSi}_{2}$ heating element. The temperature is feedback controlled to within $+/-1^{\circ} \mathrm{C}$ with programmable temperature trajectories. The furnace is mounted on a transverse horizontal-vertical stage overhanging the tomography stage to position the furnace with respect to the beam and sample, shown in figure 1a.

The sample is mounted inside of or attached to an open quartz (fused silica) tube. The sample assembly is then mounted inside a sealed quartz tube in which the sample atmosphere can be controlled. This entire assembly is inserted into the bore of the furnace using the tomography stage. Gas can then be injected into the cell to flow over or through the sample as shown in figure $1 \mathrm{~b}$. Gas flow into the environment is regulated by a mass flow controller and ambient pressure is controlled by a vacuum regulator on the outlet. For certain experiments requiring analysis of pyrolysis or other exhaust products, a residual gas analyzer can be connected to the outlet to provide a mass spectrum of the gas products.

The in-situ furnace and environmental gas cell system has been deployed at the ALS and successful $\mu$ CT analysis was performed to study high temperature processing of CMC fiber preforms for jet turbine components. Additional experiments are planned in upcoming months for CMCs and ablative heat shield materials that will utilize the full range of the furnaces capabilities. Development and testing of this furnace system have demonstrated its versatility in studying a variety of materials and process including high temperature chemical processing, pyrolysis, sintering as well as thermal decomposition processes. The furnace combined with high temperature mechanical testing capabilities provide a comprehensive suite of in-situ testing capabilities at the ALS that is well suited for analysis of aerospace materials.

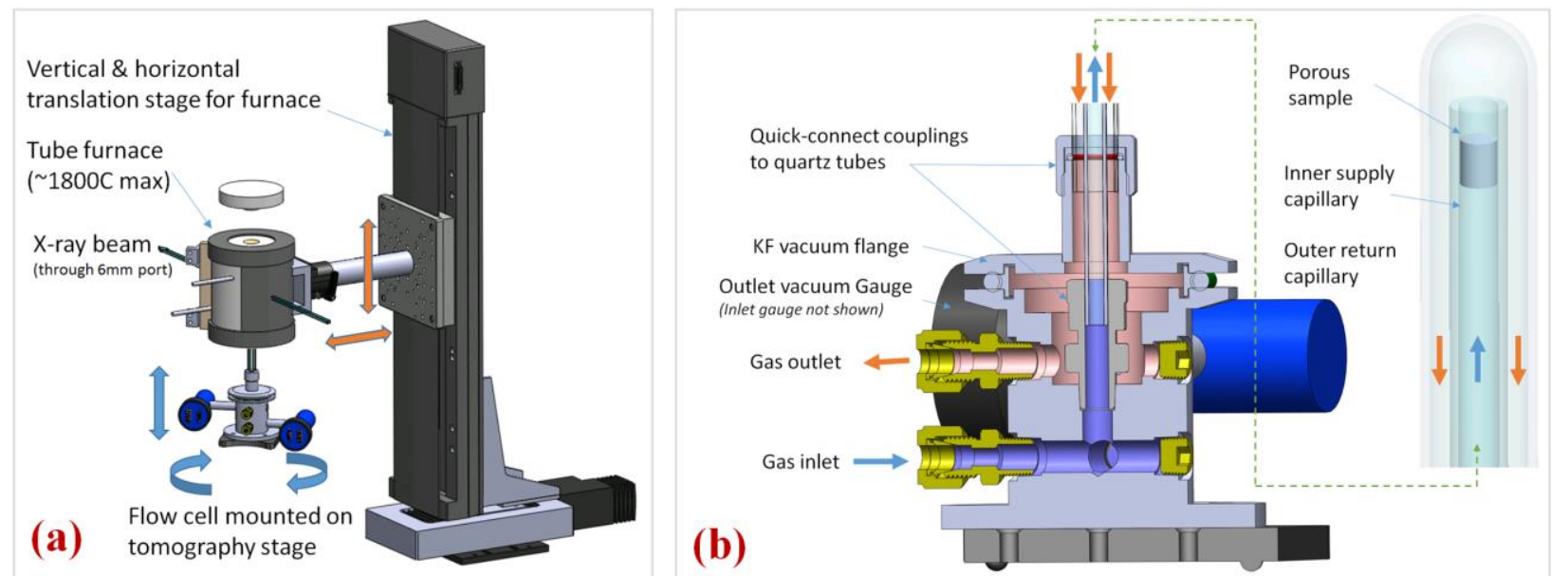

Figure 1: (a) Internal structure of high temperature environmental cell. (b) In-situ furnace system for high temperature $\mu C T$. The environmental cell is inserted into the furnace from below while the x-ray beam passes through a $6 \times 6 \mathrm{~mm}$.

[1] Naslain R, Composites Science and Technology 64 (2004): 155-170

[2] Larson N M, Zok F W, Acta Materialia 144 (2018): 579-589.

[3] Padture, N P. Nature materials 15.8 (2016): 804

[4] Panerai F, et al. International Journal of Heat and Mass Transfer 108 (2017): 801-811

[5] Venkatapathy E, et al. NASA Technical Reports Server. Thermal Protection for Mars Sample Return Earth Entry Vehicle: A Grand Challenge for Design Methodology and Reliability Verification." (2017).

[6] Barnard H S, et al. Journal of Physics: Conference Series IOP Publishing (2017): Vol. 849 No. 1.

[7] Bale H A, et al. Nature Materials 12 (2013): 40-46

[8] Haboub A, et al. Review of Scientific Instruments 85 (2014): 083702 